\title{
A method for sensation scaling based on an analogy between perception and judgment'
}

LENNART SJÖBERG

PSYCHOLOGICAL LABORATORIES, UNIVERSITY OF STOCKHOLM, SWEDEN

Turo techniques for studying judgment are discussed. In the first of these one assumes invariance of perception over different judgment processes and conceives of the properties of one judgment process as known. Then, perceptions may be estimated under this process and utilized in the study of other judgment processes of interest. A second technique disposes of the reliance up on a basic, "known" process and instead suggests that perception be treated as free parameters to be estimated from data. The paper then proceeds to show how this second technique may be used under certain conditions oy perception themselves. A concept of sensation is introduced which is thought of as generating percepts in formally the same manner as percepts are thought of as generating judgments. The idea is tried out on the perception of movement time. A simple perception process appears to give a good fit to data.

A possible way to conceive of judgment is to regard it as the outcome of a transformation on one or several percepts. In certain uses of ratio scaling methods (e.g., Stevens, 1957; Stevens \& Galanter, 1957) it is suggested, to take an example, that magnitude estimations directly reproduce ratios between perceptual quantities. Consequently, if it can be shown that the suggestion is valid, one is able to estimate scale values of percepts by means of such estimations. But there exist other ways of judging stimuli besides those utilized in scaling methods. Here it may be an entirely open question just what is the best description of the judgment transformation in question. For the case of similarity estimation of pairs of unidimensional percepts the following function has been suggested:

$$
s_{i j}=\frac{2 R_{i}}{R_{i}+R_{j}} \quad\left(R_{i}<R_{j}\right)
$$

where $s_{i j}$ is the estimated similarity on a $0-1$ scale and $\mathbf{R}_{\mathbf{i}}$ and $\mathbf{R}_{\mathbf{i}}$ are perceptual magnitudes. Equation 1 has been suggested by Eisler and Ekman (1959) and subjected to empirical test also by Eisler (1960) and Ekman, Goude, and Waern (1961). A related function has been found to hold in the multidimensional c ase (Ekman, Engen, Künnapas \& Lindman, 1964).

\section{Traditional approach to the study of judgment}

A straightforward way of investigating the validity of Equation 1 is the following: (1) Collect judgments of similarity between pairs of percepts. (2) Use some ratio scaling method to establish a scale for the stimuli used in step 1. (3) Compare empirical similarities with similarities predicted from Equation 1 and the scale values found in step 2 .

The procedure just described presupposes three things. First, the percepts involved must be the same in the scaling experiment and in the similarity estimation experiment. Let us call this condition judgment invariance of percepts. Second, there must be only one perceptual magnitude corresponding to each stimulus, i.e., regardless of what the comparison stimulus happens to be and regardless of whether the stimulus in question is standard or variable. This condition will be called context invariance of percepts. Third, the scaling method must produce a valid perceptual scale.

In some cases it may be possible to work without assuming context invariance. Equation 1 may also be written

$$
\begin{aligned}
s_{i j} & =\frac{2 q_{i j}}{1+q_{i j}} \\
\text { where } & q_{i j}=\frac{R_{i}}{R_{j}}
\end{aligned}
$$

Equation 2 may be studied directly by plotting similarities against directly estimated ratios between perceptual magnitudes, with no intervening construction of scale values. This was done by Ekman, Goude, and Waern (1961). Of course, judgment invariance and validity of the ratio estimates must still be assumed. It is, however, a restricted kind of validity where the construction of a scale is not generally possible since the construction of scales, at least in ratio estimation, presupposes at least some form of context invariance (cf., Sjoberg, 1965). If context invariance is completely given up it seems impossible to verify the assumptions behind the scaling method and consequently the investigator must accept it on rather loose grounds.

The validity of the traditional approach is, then, directly dependent on the three conditions of context invariance, a valid scaling method, and judgment invariance. These conditions will now be commented on. Context invariance and scale validity have tended to be tested jointly, so they will be treated here as one joint assumption.

Many tests have been made of direct scaling methods, using some kind of context invariance. The outcome has often been negative for the methods. For ratio estimation there is a series of studies all reporting substantial inconsistency of data matrices (Guilford, 1954; Eisler, 1960; Goude, 1962; Mashhour, 1964; Sjoberg, 1965, 1966b). For magnitude estimation a number of 
authors have reported scale distortions with a change of standard stimulus (Engen \& Levy, 1955; Stevens, 1956; Ekman, 1961). Inconsistency is by no means always found, however, and often surprising stability and generality of scales have been reported (Ekman \& Sjoberg, 1965; Goude, 1962; Stevens, 1959, 1962, 1966). It is probably fair to state that direct scaling methods may be useful under certain conditions but we have no very clear idea about just what those conditions are. Since the methods used for testing validity of the scaling method presuppose some kind of context invariance there is always the further problem of separating the effects of the two assumptions. To date, very little progress has been made with this problem (cf., Sjoberg, 1965, 1966b).

As for the assumption of judgment invariance, it seems very difficult to say anything certain. One can only speculate in the possibilities of perception changes as a function of the judgment process. Such changes could start even at the peripheral level (Livingstone, 1959; cf., Eisler, 1965).

In summary, the approach to the study of judgment outlined in this section presupposes: (1) Judgment invariance of percepts. (2) Context invariance of percepts. (3) Validity of the scaling method used.

\section{Alternative approach to the sfudy of judgment}

The validity of a certain postulated form of the judgment process, like Equation 1, may be studied without a separate scaling of the perceptual magnitudes involved. If, for example, judgments of similarity are collected for all the possible $n(n-1) / 2$ pairs, given $n$ stimuli, these pairs generate $n(n-1) / 2$ equations like Equation 1. The number of unknowns is $n-1$, since we have $\mathrm{n} R$-values and an arbitrarily specified unit. Consequently the system is overdetermined and goodnessof-fit tests may provide information as to the validity of Equation 1. This is essentially the same procedure as when the "internal consistency" of data used for scaling is studied.

The goodness-of-fit test presupposes at least some kind of context invariance; or we get more unknowns than equations. The procedure has two advantages over the traditional approach: it does not assume judgment invariance of percepts and it does not presuppose the validity of any scaling method. It does, however, assume context invariance of some kind. (The formal function of the assumption of context invariance is to save a certain number of degrees of freedom so as to make a goodness-of-fit test possible.) It may also be less powerful than the traditional approach when the assumptions of judgment invariance and validity of the scaling method both hold. This is because the $R$-values are treated as free parameters, which makes it more difficult to disconfirm any given postulated judgment equation (R-values are "allowed" to take their values so as to make up for inadequacies of the postulated equation).
The approach has been tried out on Equation 1 under two conditions of context invariance (Sjoberg, 1966a):

Condition 1. Only one $\mathrm{R}$-value corresponding to each stimulus, i.e., complete context invariance.

Condition 2. Two R-values corresponding to each stimulus: one when it appeared as the largest stimulus in a given pair and one when it appeared as the smallest stimulus in a given pair.

Four sets of previously published data were studied (the continua of pitch, heaviness, greyness and circular area). In all cases, except possibly that of pitch, it was clear that Condition 1 and Equation 1 tested jointly did not fit. When Condition 1 was replaced by Condition 2 the fit was much improved and no systematic errors could be detected. Similar findings are reported in a later study of circular area and heaviness (Sjöberg, 1966b).

As already mentioned, much work has been done with the "internal consistency" of data used for scaling purposes. It is interesting to note that much of this work is conducted within a frame of reference that is essentially the same as the present approach to the study of judgment generally. Thus, Condition 2 above is a form of context invariance that has given some promise in the study of direct scaling methods at least as regards the description of "inconsistency" (Sjöberg, $1965,1966 \mathrm{~b})$.

\section{Sensation scaling}

We have treated judgment as a transformation of percepts. The basic idea of the method of sensation scaling to be proposed is to treat perception itself as a transformation of some basic sensory "input," here for convenience called "sensation." Sensation as conceived here need not be made conscious in order to be studied; instead it is scaled indirectly, using properties of percepts.

Perhaps the idea can be illustrated with the sizeweight illusion. Here we have an interaction of visual and kinesthetic input which produces a final percept of heaviness which depends upon both. What is here called the perception process is exactly that interaction. The sensory input, visual and kinesthetic, that enter the perception process, is what we call sensation. From a purely formal point of view, the situation is entirely analogous with judgment, where percepts enter a transformation process which eventually yields an overt response as its final product.

In judgment, two alternative ways of conducting a study have been discussed. One might ask now if similarly we have two ways of studying sensationperception. The traditional approach to the study of judgment would correspond to attempts of making sensations conscious, as well as percepts, and then try to predict percepts from sensations. Something like this was tried early in the history of psychology (Wundt, Titchener) but that approach does not receive much attention now. Presumably this is because it seems 
difficult to make sensations conscious without at the same time turning them into percepts. At any rate it is hard to demonstrate that the procedure really yields a true picture of sensation. The scaling method advoated here proceeds as the alternative way of studying judgment.

First of all it is necessary to have a valid scale of the percepts involved. This is the starting point for our analysis. In the following we will simply assume that il scale of percepts has been constructed. The reader who believes the construction of such scales to be impossible will have little further interest in this paper.

Let us return for a moment to the size-weight example. We decide to use, say, 8 different weights and 7 different sizes. Stimulus objects are then constructed in all possible ways ( 56 , or $7 \times 8$ ). We establish scale values for all 56 objects by some suitable psychophysical procedure, e.g., magnitude estimation. The subjects are simply instructed to report perceived heaviness, and nothing else, just as in any other psychophysical experiment.

We now make a critical assumption about sensation invariance, i.e.s that each weight, and each size, gives rise to a constant sensory input regardless of which particular stimulus object it occurs in. We thus assume size 2 in our example to produce the same visual input regardless of the physical weight of the stimulus object in which it occurs, to take an example. Utilizing this assumption we need only introduce one unknown parameter for each sensation value. Thus we get 8 unknown sensation values for weight and 7 unknown sensation values for size. The perceived heaviness of a particular stimulus object then depends on (1) the two relevant sensation values and (2) the exact form of the interaction between visual and kinesthetic input in producing the final product.

More concretely, the idea involves setting up an overdetermined system of equations where each scaled stimulus object gives rise to one equation. The unknowns are the sensation values and the form of the equation is dictated by the nature of the perception process concerned. Goodness-of-fit tests may be used to indicate the validity of the assumptions of sensation invariance and the particular form of the equations.

The technique is here developed only for cases of more than one sensation dimension. It might be argued that in such cases percepts can be expected to be multidimensional, but the approach here is to conceive of percepts as unidimensional and sensation as multidimensional. Just how reasonable it is to expect unidimensional percepts in spite of multidimensional sensation is of course an empirical question.

\section{The requirement of sensation invariance and stimulus description problems \\ A :iven stimulus object can of course be described equally exhaustively in a very large number of ways.}

Only some of these descriptions are relevant in sensation scaling, viz. those that tend to produce a very high correlation between physical values and sensation values. The physical attributes of such good descriptions will be called "cues." Naturally, what is a good description for one postulated form of the perception process may be bad for another.

Thus, the requirement of sensation invariance may be impossible to meet with a particular physical description of stimuli and a particular equation. A good cue hypothesis is always necessary for sensation scaling, as conceived here.

Proximal stimulus description appears to have better chances of achieving sensation invariance than distal description. Perfect invariance may not be expected under any circumstances, because of inherent variability of the receptor system.

\section{Processes in the perception of velocity and movement time}

Brown $(1931 \mathrm{a}, \mathrm{b})$ raised the question of the relations between phenomenal time, phenomenal velocity and phenomenal distance. He suggested the equation

$$
R_{v}=R_{d} / R_{t}
$$

where $R_{v}$ is phenomenal velocity, $R_{d}$ is phenomenal distance and $\mathbf{R}_{\mathbf{t}}$ phenomenal time. As pointed out by Mashhour (1964) Brown's own experiments could not demonstrate the validity of Equation 3. Mashhour performed an experiment where all three variables were scaled and found that Equation 3 was false.

Other recent studies have been reported by Bonnet (1964) and Rachlin (1966). Bonnet did not use scaling procedures. Rachlin, however, used methods similar to Mashhour's and also found Equation 3 to be wrong. Furthermore, Rachlin reported strong effects on judgments of movement time from changes in velocity and distance, just as Mashhour.

The kind of experiment performed by Mashhour and Rachlin tells us nothing directly about the process of velocity perception as conceived here. We would instead postulate the sensation attributes of distance and time as determiners of velocity perception. Stimulus objects would be defined by different combinations of time and distance.

Assuming sensation invariance and using $\mathrm{C}_{\mathrm{d}}$ for a distance sensation value and $C_{t}$ for a time sensation value we can postulate the process

$$
R_{v}=C_{d} / C_{t}
$$

where $R_{v}$ is our measure of subjective velocity. If we introduce necessary arbitrary scale units we see that Equation 4 implies that each $R_{\mathrm{v}}$ can be interpreted as a ratio multiplied by a certain constant. Thus Equation 4 can easily be subjected to empirical test by scaling 


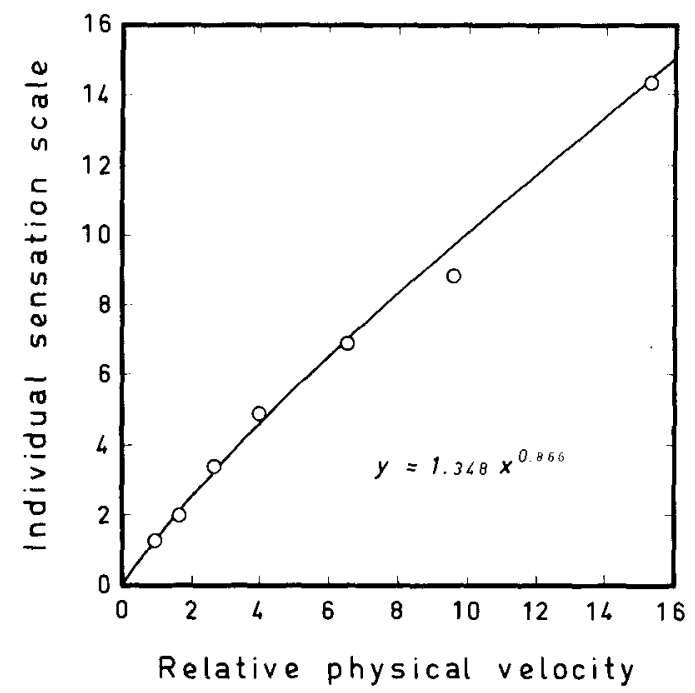

Fig. 1. Sensation scale for a single individual against stimulus velocity.

the perceived velocities of a number of stimuli generated by suitable combinations of movement time and distance.

The related problem of movement time perception is to be empirically approached in this paper. Equations 3 and 4 suggest a mechanism for time experiences, generated by the stimulus dimensions velocity and distance travelled. We have

$$
\mathrm{R}_{\mathrm{t}}=\mathrm{C}_{\mathrm{d}} / \mathrm{C}_{\mathrm{v}}
$$

with designations analogous to those of Equation 4. It is tempting to formulate a third equation

$$
\mathbf{R}_{\mathrm{d}}=\mathrm{C}_{\mathrm{t}} / \mathrm{C}_{\mathrm{v}}
$$

and study the connections between Equations 4-6. Such an approach will not be pursued here, since data only allow for the direct study of Equation 5. In addition, even if all three Equations 4-6 turned out to hold empirically, there is no guarantee that the exact form of the sensation scales would be the same.

Table 1. Mashhour's median subjective estimates of perceived time for a group of subjects.

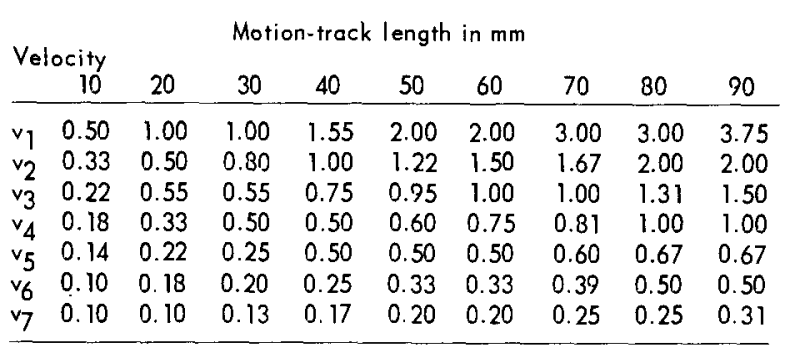

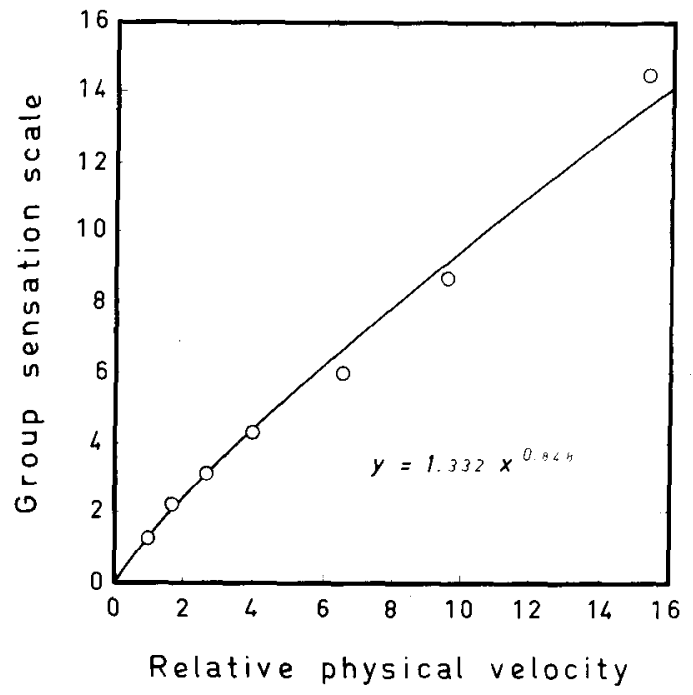

Fig. 2. Sensation scale for a group against stimulus velocity.

\section{Empirical application}

Mashhour's experiment resulted in some data on magnitude estimation of time that will be appropriate for a test of Equation 5. As stimuli Mashhour used small moving spots of light moving horizontally on an oscilloscope screen. He used all the 63 stimulus conditions resulting from all the possible combinations of 7 velocities and 9 distances. Data are given both for a group of 30 subjects (medians will be used) and for a single subject who made seven ratings of each stimulus (here we use the arithmetic means). Tables 1 and 2 give the data that are analyzed in this paper.

Since Mashhour's experiment generated an entire matrix here interpreted as a matrix of ratios according to Equation 5 component analysis is a good analytical method for testing the equation and estimating the sensation values (see Gulliksen, 1959, for illustrations of component analysis). The data matrices of time ratings should be of rank one. Table 3 gives an account of their characteristic values, clearly indicating a good approximation to rank one.

Figures 1 and 2 present the sensation values of velocities against their corresponding physical values.

Table 2. Arithmetic means of estimates of perceived time from a

\begin{tabular}{|c|c|c|c|c|c|c|c|c|c|}
\hline & & & Mo & -tra & ens & $\mathrm{m}$ & & & \\
\hline & $\begin{array}{c}\text { city } \\
10\end{array}$ & 20 & 30 & 40 & 50 & 60 & 70 & 80 & 90 \\
\hline$v_{1}$ & 0.44 & 0.80 & 1.16 & 1.66 & 2,03 & 2.36 & 3.07 & 3.36 & 3.50 \\
\hline$v_{2}$ & 0.35 & 0.49 & 0.91 & 0.89 & 1.29 & 1.51 & 2.01 & 2.07 & 2.21 \\
\hline$v_{3}$ & 0.26 & 0.34 & 0.54 & 0.66 & 0.87 & 1.01 & 1.04 & 1.29 & 1.19 \\
\hline$v_{4}$ & 0.16 & 0.29 & 0.38 & 0.43 & 0.50 & 0.65 & 0.30 & 0.91 & 0.91 \\
\hline$v_{5}^{4}$ & 0.15 & 0.24 & 0.24 & 0.37 & 0.37 & 0.46 & 0.55 & 0.61 & 0.63 \\
\hline$v_{6}$ & 0.10 & 0.15 & 0.19 & 0.23 & 0.31 & 0.34 & 0.39 & 0.54 & 0.50 \\
\hline $\begin{array}{r}0 \\
v_{7}\end{array}$ & 0.07 & 0.10 & 0.13 & 0.20 & 0.22 & 0.23 & 0.28 & 0.25 & 0.29 \\
\hline
\end{tabular}
single subject from Mashhour. 


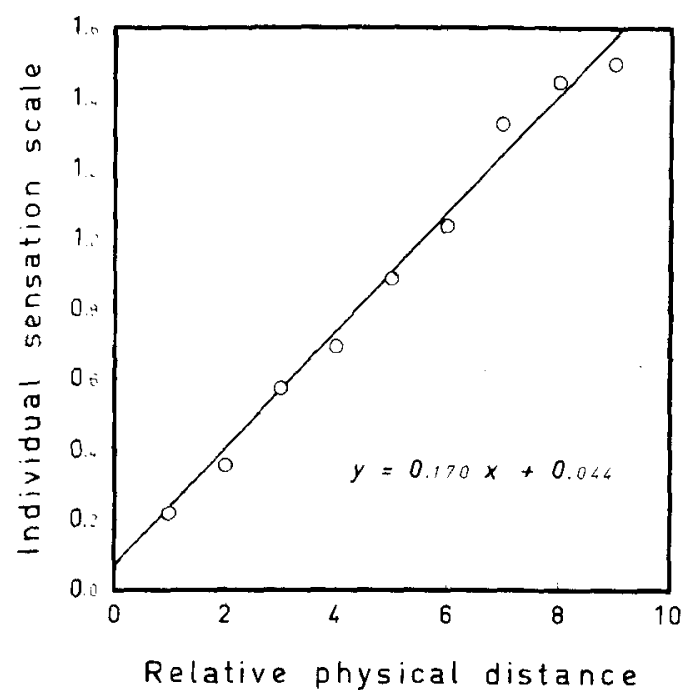

Fig. 3. Sensation scale for a single individual against stimulus distance.

Two very similar power functions have been fitted, both with exponents about .85. The corresponding plots for distances appear in Figs. 3 and 4. Straight lines have been fitted, both yielding positive intercepts.

\section{Discussion}

The empirical findings reported are somewhat difficult to evaluate. Equation 5 gave a very good fit to data, as witnessed by Table 1 . The sensation scales for distance were quite similar to independently derived perception scales, but for velocity there was a discrepancy between sensation and perception. On the other hand, Mashhour himself has reported negatively accelerated power functions for perceived velocity as a function of physical velocity (Mashhour, 1964). Rachlin (1966) reported exponents about 0.75 for judgments of linear velocity. Neither similarity nor difference between sensation and perception scales constitutes conclusive evidence of the validity of any of the measures used, of course. Some attention has been paid to such comparisons here because the technique of sensation scaling was entire.y new and no sensible predictions about the quantitative properties of "sensation, as

Table 3. Characteristic values of the two matrices of movement time scales.

\begin{tabular}{ccc} 
Value no. & Group data & Individual data \\
\hline 1 & 76.66 & 79.94 \\
2 & 0.29 & 0.08 \\
3 & 0.07 & 0.05 \\
4 & 0.03 & 0.02 \\
5 & 0.01 & 0.01 \\
6 & 0.00 & 0.00 \\
7 & 0.00 & 0.00 \\
\hline
\end{tabular}

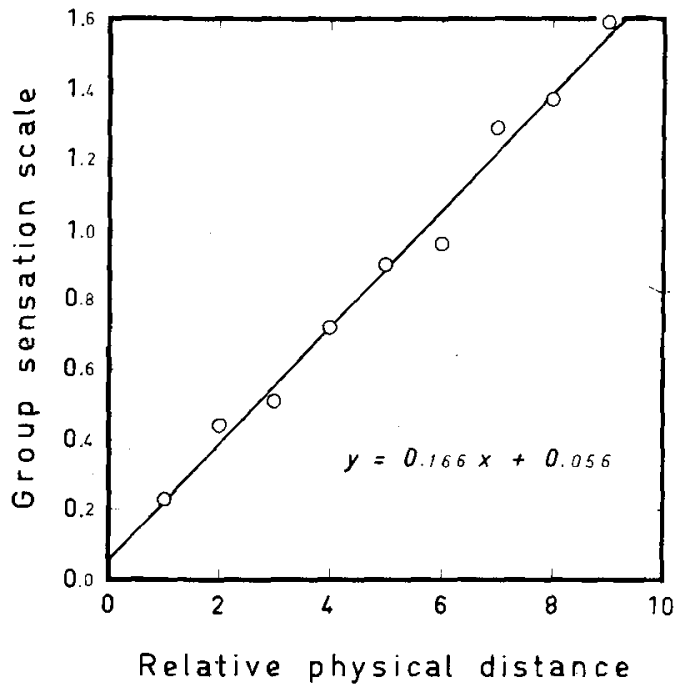

Fig. 4. Sensation scale for a group against stimulus distance.

different from perception, could be made.

A more important reason for sensation-perception comparisons is this. Consider the case of distance. If it is directly estimated we get a linear $\mathrm{R}-\mathrm{S}$ function with a positive intercept. A very similar function can be fitted to sensation values derived from time estimations. This would seem to be an interesting discovery of a physical continuum having very similar effects in two widely different experimental situations. This is the kind of empirical phenomenon that may be accounted for and discovered with the use of a concept of sensation such as the present one.

The total process from stimulus to response involves the third part of judgment besides the first two processes of sensation and perception. Some readers might consider it advantageous to deal with the whole sensation-perception-judgment complex in one single context. This paper has treated sensation-perception and perception-judgment separately. This introduces some simplicity both for purposes of exposition and research. The risk of oversimplification has admittedly been taken but it may, perhaps, be argued that the risk appears to be no larger here than in many other kinds of psychological methodology.

\section{References}

Bonnet, C. La vitesse percue et la relation $\mathrm{V}=\mathrm{E} / \mathrm{T}$. Ann'c' $p s^{\prime} y^{-}$ chol., 1964, 64, 47-60.

Brown, J. F. Visual perception of velocity. Prychol. Forsch., 1931a, 14, 199-232.

Brown, J. F. On time perception in visual movement fields. Psychol. Forsch., 1931b, 14, 233-248.

Eisler, H. Similarity in the continuum of heaviness with some methodological and theoretical considerations. Stand. J, Pryl- 
i'hol., 1960, 1, 69-81.

Hisler, It. On psychophysies in general and the general psychophysical differential equation in particular. Scand. J. Psychol., $1965,6,85-102$.

Eisler, H., \& Ekman, G. A mechanism of subjective similarity. Acta psychol., Amsterdam, 1959, 16, 1-10.

Fkman, G. Methodological note on scales of gustatory intensity. Scand. J. Psuchol., 1961, 2, 185-190.

Ekman, G., Engen, T., Künnapas, T., \& Lindman, R. A quantitative principle of qualitative similarity. J. exp. Psychol., 1964, 68, 530-536.

Ekman, G., Goude, G., \& Waem, Y. Subjective similarity in two perceptual continua. J. exp. Psychol., 1961, 61, 222-227.

Ekman, G., \& Sjoberg, L. Scaling. Annu. Rev. Psychol., 1965, $16,451-474$.

Engen, T. \& Levy, $\mathbf{N}$. The influence of standards on psychophysical judgment. Percept. mot. Skills, 1955, 5, 193-197.

Goude, G. On fundamental measurement in psychology. Stockholm: Almqvist \& Wiksell, 1962.

Guilford, G. Psychometric methods. New York: McGraw-Hill, 1954.

Gulliksen, H. Mathematical solutions for psychological problems. Amer. Scientist, 1959, 47, 178-201.

Livingstone, $\mathbf{R}$. Central control of receptors and sensory transmission systems. In J. Field, H. Magoun, \& W. Hall (Eds.), Handbook of physiology. Section I: Neurophysiology. Vol. I. Washington, D. C.: American Physiological Society, 1959.

Mashhour, M. Psychophysical relations in the perception of velocity. Stockholm: Almqvist \& Wiksell, 1964.
Rachlin, H. C. Scaling subjective velocity, distance, and duration. Purcept. \& Psychophys. 1966, 1, 77-82.

Sjöberg, L. On ratio estimation. Report Psychologjcal Laboratory, University of Stockholm, No. 191, 1965.

Sjoberg, L. Unidimensional similarity revisited. Scand. J. Psychol., 1966a, 7, in press.

Sjoberg, L. A comparative study of three direct psychophysical scaling methods. Unpublished manuscript, $1966 \mathrm{~b}$.

Stevens, S. S. The direct estimation of sensory magnitudes-lind. ness. Amer. J. Psychol., 1956, 69, 1-25.

Stevens, S. S. On the psychophysical law. Psychol. Rev., 1957, $64,153-181$.

Stevens, S. S. Cross-modality validation of subjective scales for loudness, vibration, and electric shock. J. exp. Psychol., 1959. 57, 201-209.

Stevens, S. S. The surprising simplicity of sensory metrics. Amet. Psychologist, 1962, 17, 29-39.

Stevens, S. S. Matching functions between loudness and ten other continua. Percept. \& Psychophys., 1966, 1, 5-8.

Stevens, S. S., \& Galanter, E. H. Ratio scales and category scales for a dozen perceptual continua. J. exp. Psychol., 1957, 54, 377-411.

\section{Note}

1. The author gratefully acknowledges valuable comments on this paper by Drs. Mats Björkman, Hannes Eisler, Gösta Ekman, Gunnar Goude, William Lambert and Ingvar Lundberg.

(Received in the Editorial office March 28, 1966.) 